\title{
Editorial: Mahjong, Sudoku and Chess
}

\author{
Tristan Cazenave \\ LAMSADE, Université Paris-Dauphine, PSL, CNRS, Paris, France \\ E-mail:Tristan.Cazenave@dauphine.psl.eu
}

My first words go to Professor Mark Winands. Thank you Mark for your dedication to the ICGA and for your excellent work as acting Editor-in-Chief during the last two years. I will try my best to match the high standards you and Professor I-Chen Wu have set.

This first issue of 2021 contains two scientific articles. The first one An Efficient AI-based Method to Play the Mahjong Game with the Knowledge and Game-tree Searching Strategy by Mingyan Wang et al. deals with the imperfect information game of Mahjong which has a huge average size of information sets. In order to approach this popular, complex and challenging game, the authors propose to use opponent models, knowledge and tree search.

The second article is Sudoku Ripeto. A Variant of Sudoku with Repeated Symbols by Miguel G. Palomo. He defines some Sudoku Ripeto families, with different repetition patterns and gives algorithms using rules and search to complete squares. He also analyzes some rules that can be used to solve puzzles and gives sufficient conditions for puzzles to be flexibly solved without search.

In the Book reviews section, Guy Haworth gives us a review of the $5^{\text {th }}$ edition of the late Mark Dvoretsky's Endgame Manual.

In the News section, Monty Newborn proposes a new variant on the game of Chess and Chess960 named Mad Monty Chess and GM Matthew Sadler analyzes some Chess960 games that were played at the Top Chess Engine Championship. Guy Haworth and Nelson Hernandez report on the 20th Top Chess Engine Championship, TCEC20 and GM Matthew Sadler gives us a perspective on the TCEC20 Computer Chess Superfinal. You will also find a report on the TCEC Cup 8 as well as the SSDF Chess Engine Rating List.

Tristan Cazenave 